\title{
Numerical studies of steel pipe sheet pile
}

\author{
Vadim Alpatov ${ }^{1, *}$ and Alexandre Bezuglov ${ }^{1}$ \\ ${ }^{1}$ Samara State Technical University, Academy of Architecture and Civil Engineering, \\ Molodogvardeyskaya St., 194, Samara, 443001, Russia
}

\begin{abstract}
The sheet pile wall (SPW) is carried out from pipes of big diameter which plunge into soil very deeply. Depth of pipes is the settlement size depending on a set of parameters. Depth of pipes provides rigidity and reliability of a construction. SPW has a high rate of a consumption of steel on his production. Authors of the present article suggest to reduce a material capacity of SPW at the expense of reduction of depth of blockage of every second pipe. Decrease in depth of blockage of separate pipes is carried out at preservation of the maximum depth of blockage of other pipes. The design similar to a «comb» because of alternation of long and short pipes turns out. Such decision allows to reduce the total depth of blockage of pipes and as a result leads to decrease in a material capacity and cost of a construction in general. The problem of search of possible reduction of length of a part of pipes is the purpose of the real research. As solutions of the put problem we suggest to execute numerical experimenting in iterative statement with use of modern software. Results of numerical researches of SPW are presented in the present article. The presented results allow to draw a conclusion on efficiency of the solution on decrease in a material capacity of SPW proposed by authors. For obtaining the generalized decision and obtaining undoubtedly the best result of a design of SPW authors consider necessary to execute problem definition in the form of parametrical optimization.
\end{abstract}

\section{Introduction}

With the evolution of the market economy and the transition of the construction industry from costly management methods to the replacement of resource- consuming and expensive retaining walls of gravitational type, made from natural stone, monolithic and prefabricated reinforced concrete, thin-walled structures of various constructions with steel piles were developed. Steel pile has a potential for development because of number of advantages in compare with similar, but more time-consuming and complex solutions. The development of the application with SPW in transport, hydraulic engineering and special construction began in the late 1980 [1]. The advantages of a steel pipe in front of other types of pipe is well-known [2-4]. A feature of this design is the inability to apply a sparse or unequal pitch sheet. For SPW it is typical that on the entire length of the wall the piles are made of the same diameter and are arranged with an equal pitch. Step piles depends on the accepted diameter of the pipe and the dimensions of the lock. Usually, SPW is performed with the

*Corresponding author: avu75@mail.ru 
penetration of each pile on the same depth (Figure 1). This design is characterized by uniform operation and equal reliability in the entire perimeter of the structure [5-9]. For this design, methods for estimating its load capacity and rigidity were developed.

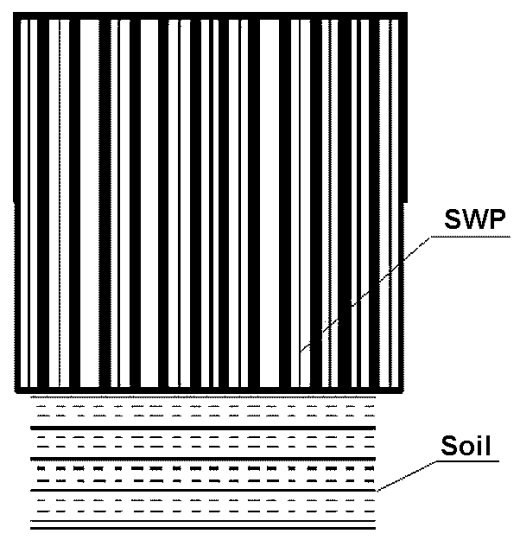

Fig. 1. Scheme of SPW construction.

Pipes with large diameter and depth of piling are used in the construction of tubular tongue. The cost of the device is significant, including increasing consumption of metal on a continuous deep steel dowel. Dense hammering sheet pile is caused by the technology of SPW. The depth of the face is a design value that depends on a number of parameters and rigidity and reliability of the structure. SPW has a high rate of steel consumption for its production. Researches [10] show that pipes in the SPW often operate with a large margin of bearing capacity and have a significant margin of rigidity.

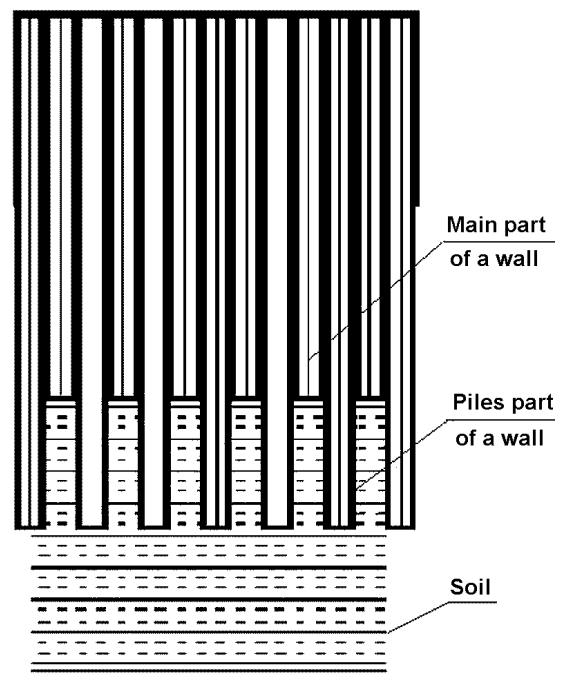

Fig. 2. Scheme authors' construction of SPW - «comb».

The authors of this article suggest to reduce material consumption of the SPW by reducing total reduced depth of plowing. It means a decrease in the depth of the piling of a part of the piles while maintaining the maximum depth of piling remaining. In practice, such a proposal looks like an alternation of long and short dowels, forming a kind of "comb" (Figure 2). Such a solution allows to reduce the overall depth of piling and, as a consequence, leads to a decrease in the material capacity and the cost of the whole 
structure. The problem of finding the possible reducing the length of the section of the tongue without losing the reliability of the structure, is the purpose of this research. As a way to solve the problem, numerical experimentation in iterative setting using modern software was adopted (Figures 3-6) [11, 12].

\section{Materials and methods}

In order to reduce the final cost of one ton of products "in works", the pile wall system consisting of pipes without the use of gills piles with constant length (Figure 1) is proposed to be changed. It was decided to give the wall a comb-like shape, using the alternating length of the piles (Figure 2). The proposed solution by the authors to reduce the cost of construction and installation works for the building of structures from SPW due to the reduction of the material capacity of the large-diameter steel pipes required a number of researches to verify the possibility of obtaining a positive result.

The purpose of the research was to assess the feasibility of comb-like construction of the SPW without reducing its reliability and safety.

Choosed method of research - numerical iteration modeling of a comb construction of SPW.

The execution tool for researchers was the MidasGTSNX software environment. It is customary to use the capabilities of the MidasGTSNX 2015 for verification. The complex allows to simulate the site conditions including nonlinearity of the material and the initial stresses in the ground. MidasGTSNX supports all types of calculations including static, dynamic, calculation of filtering, combined calculation of VAT and filtration, consolidation, calculation taking into account the sequence of construction, as well as calculation of stability.

During design of a calculating model the following constraints were accepted:

1. The system "SPW-soil mass" was modeled (Figure 3).

2. Only the assortment of pipes of welded electrically welded pipes was considered.

3. Free height of the wall was 6.0 meters.

4. For the initial settlement situation, the SPW design (with the same length of all piles) was adopted, satisfying all the requirements of the standards (in strength and deformation). The subsequent iterations contained calculations with a step-by-step reduction in the length of each second pile. The size of the comb part SPW varied from 0 to 6 meters in increments of $1.0 \mathrm{~m}$.

5. The same ground conditions were used for all iterations.

6. The same maximal depth of hammering the pipes was used for all iterations. Reducing the length of hammering of pieces of pipes was carried out with the same step from iteration to iteration.

7. As a result of calculations, maximum displacements, maximum stresses in the ground and in the pipes were monitored.

Selected results of calculations of several models are presented in Figures 4 and 5. The authors plotted the horizontal displacements of the tongue as a function of the size of the sparse part of the comb (Figure 6). Analysis of the calculation results of models showed that the use of alternating piles of different length in the SHS reduces the rigidity of the structure and causes a redistribution of flexural stresses along the height of the tongue. Decrease in rigidity occurs after a decrease in the length of short piles of the "crested" SPW, at the same time there is an increase in stresses in steel piles. The character of changes in rigidity is non-linear. With a step-by-step reduction in the length of the short part of the pile, there is an uneven increase in horizontal displacements, and under certain conditions, a slight decrease. Such a change in the displacements leads to conclusions about the possible existence of an optimal geometry of the "comb" dowel, which has sufficient 
rigidity and low steel consumption. The change in flexural stresses in SPW with the variation in the length of the short part of the tongue, together with the known significant safety factor of the structure, also allows us to reflect on the formulation of the problem of searching for a rational design of SPW possessing sufficient strength and reliability and low steel consumption $[13,14]$.

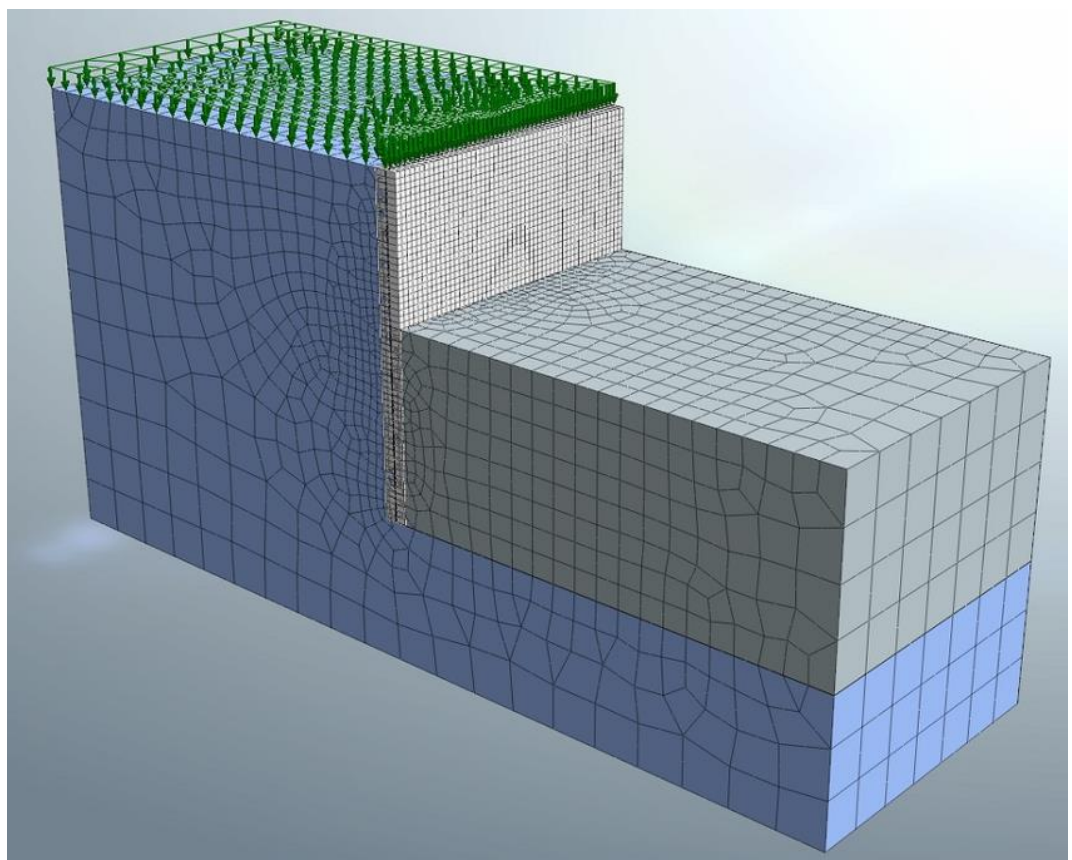

Fig. 3. Calculating 3-D model of SPW.
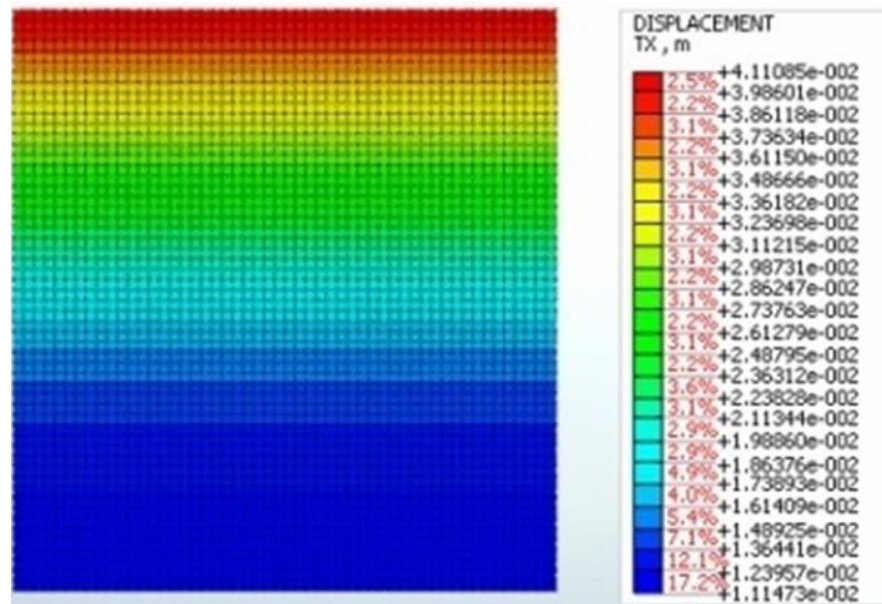

Fig. 4. Movement in SPW. 


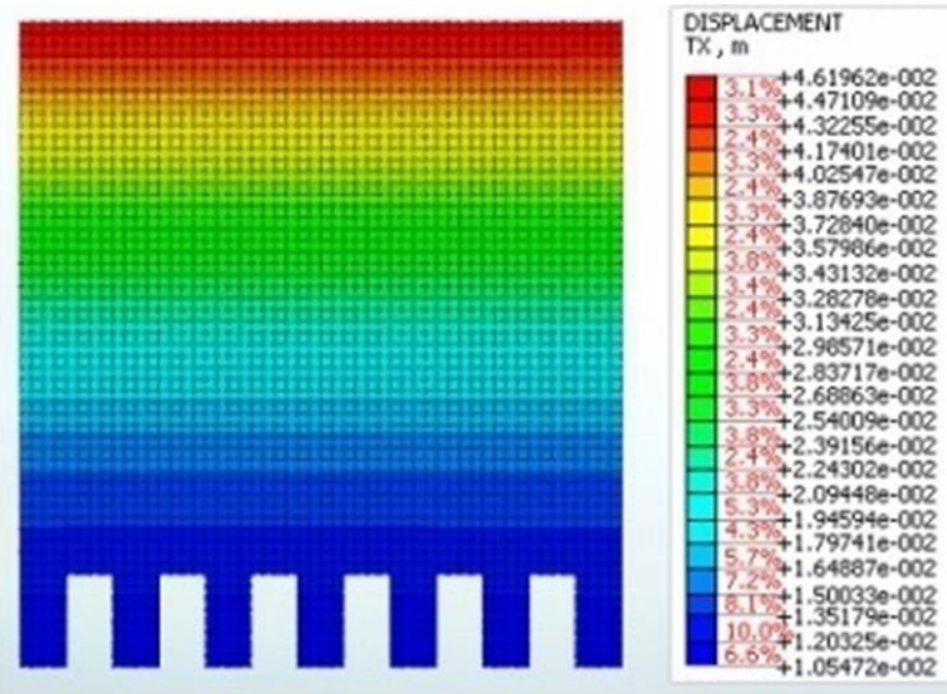

Fig. 5. Movement in SPW with depth of "comb" equals 2 meters.

105

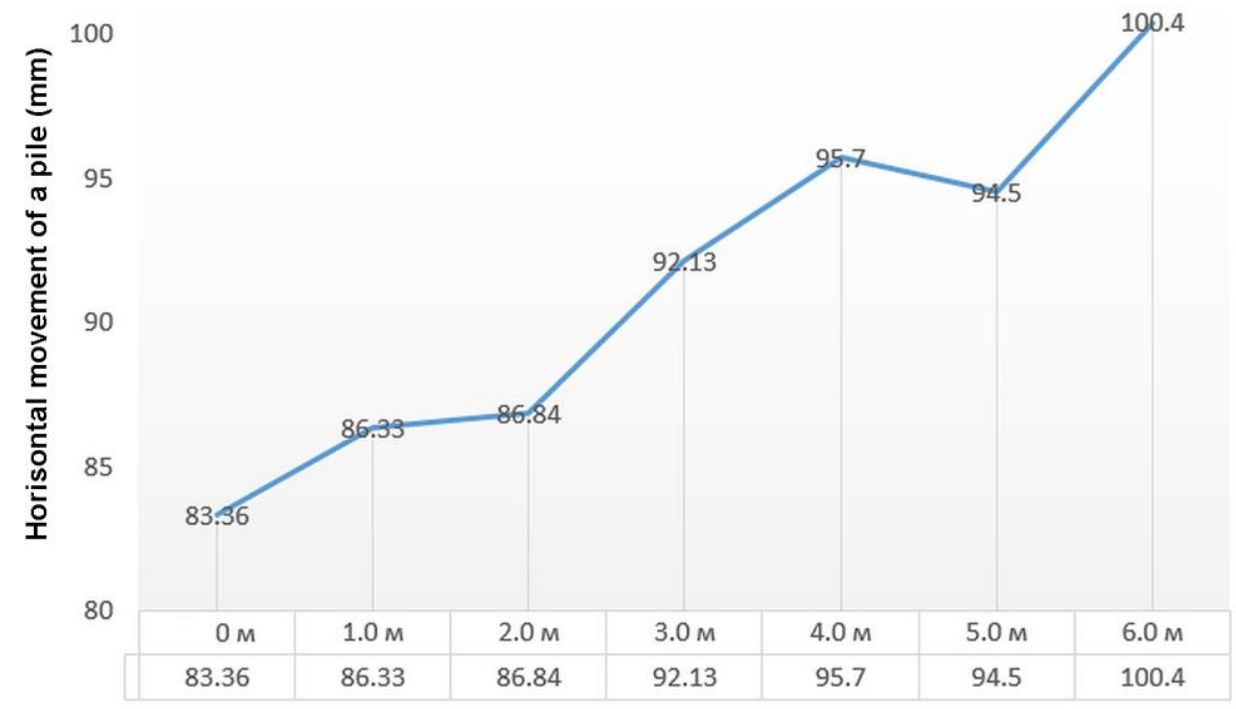

Fig. 6. Movement in SPW with changes in characteristics of «comb».

\section{Conclusions}

In this article the results of the numerical analysis of a comb SPW are shown. This results allow us to make conclusions that:

1. The proposed solution of reducing material-consuming of SPW by interlacing long and short piles showed it's effectiveness; 
2. Numerical researches showed a non-linear character of the change in the general characteristics of the rigidity of the grooved comb wall, depending on the length of the short part of tongue;

3. Performing iterative recalculations in specialized software complexes has a number of drawbacks, they are the greater complexity of the process, the large time costs for obtaining a solution, the solution for only a particular case with a certain set of initial parameters, ambiguity in determining the best result;

4. In order to obtain a generalized solution and the indisputably better result of the design of the SPW, the authors consider that it is necessary to perform the formulation of the problem in the form of parametric optimization. The formulation and solution of such a problem will avoid obtaining doubtful results because it is known that due to the peculiarities of parametric optimization problems the solution obtained corresponds to a local or global extremum within the limits of the imposed restrictions

\section{References}

1. V.N. Udovichenko, Marine and river hydraulic structures (Moscow: Transport) (1976)

2. V.E. Darevskiy, Structures that hold soil (Moscow: Master) (2011)

3. D.S. Konyuhov, The use of underground space (Moscow: Architecture-S) (2004)

4. G.S. Fokin, Metal structures and their installation (Kharkiv: East regional center for humanitarian and educational initiatives) (2006)

5. M. Kalmova, A. Mumortsev, et al, Technical mechanics (Samara: Samara state University of architecture and construction) p 144

6. V. Alpatov, MATEC Web Conf. 8602005 (2016)

7. V. Alpatov, A. Soloviev, MATEC Web of Conferences 19601008 (2018)

8. V. Zubkov, A. Lukin, et al, MATEC Web of Conferences 19601005 (2018)

9. I. S. Kholopov, et al, Building materials, equipment, technologies of XXI century 1, pp. 66-68 (2008)

10. A.A. Bezuglov, Traditions and innovations in construction and architecture. Construction, pp. 55-59 (2016)

11. A. Lukin, I. Kholopov, et al, Procedia Engineering 153, pp. 1877-7058 (2016)

12. Y.S. Nayshtut, Traditions and innovations in construction and architecture. Construction, p. 56 (2013)

13. V.Yu. Alpatov, I.S. Kholopov, Metal construction 1, pp. 47-57 (2009)

14. A.A. Bezuglov, et al, Industrial and civil engineering 9, pp. 73-76 (2017)

15. I.S. Kholopov, M. I. Balzannikov, et al, Procedia Engineering 153, pp. 277-282 (2016) 